\title{
ON THE COMPLETE INDEPENDENCE OF THE POSTULATES FOR BETWEENNESS*
}

\author{
BY
}

\author{
W. E. VAN DE WALLE
}

The paper on betweenness published by Huntington and Kline in $1917 \dagger$ contained eleven sets of independent postulates, selected from a basic list of twelve postulates, as follows:
(1) A, B, C, D, 1,2;
(7) A, B, C, D, 2,5;
(2) A, B, C, D, 1,5;
(8) A, B, C, D, 3,5;
(3) $\mathrm{A}, \mathrm{B}, \mathrm{C}, \mathrm{D}, \quad 1,6$;
(9) $\mathrm{A}, \mathrm{B}, \mathrm{C}, \mathrm{D}, \quad 3,4,6$;
(4) A, B, C, D, 1,7;
(10) A, B, C, D, 3,4,7;
(5) A, B, C, D, 1,8;
(11) A, B, C, D, $3,4,8$.
(6) $\mathrm{A}, \mathrm{B}, \mathrm{C}, \mathrm{D}, \quad 2,4$;

The purpose of the present paper is to exhibit the "complete existential theory" (in the sense of E. H. Moore + ) of each of these sets. This requires the discussion, in the usual way, of $2^{6}=64$ examples for each of the sets (1)-(8), and $2^{7}=128$ examples for each of the sets (9)-(11).

The results show that sets (1)-(10) are completely independent while set (11) is not.

In the case of sets (1), (2), (3), (5), (6), (7), which happen to be the sets which do not contain either postulate 3 or postulate 7 , the necessary examples are given in terms of a class $\mathrm{K}$ containing only four elements.

In the case of sets (4), (8), (9), (10), and (11), some of the examples require the use of a class $K$ containing five elements. These five-element

- Presented to the Society, March 1, 1924.

†E. V. Huntington and J. R. Kline, Sets of independent postulates for betweenness, these Transactions, vol. 18 (1917), pp. 301-325. For a twelfth set of postulates, which need not here be considered, see E. V. Huntington, $A$ new set of postulates for betweenness with proof of complete independence, in the present number of these Transactions.

$\ddagger$ For a discussion of the significance of "complete independence", with bibliographical references, see the paper by E.V.Huntington, in the present number of these Transactions. 
examples are used, however, only in cases where an exhaustive examination has shown that no four-element example with the same record exists.

The failure of set (11) to be completely independent is due to the nonexistence of only two examples, ${ }^{*}$ namely an example satisfying postulates $\mathrm{A}, \mathrm{B}, \mathrm{C}, \mathrm{D}$ and 8 , and violating postulates 3 and 4 ; and a corresponding example violating $\mathrm{D}$.

Table I defines 294 systems $(\mathrm{K}, \mathrm{R})$ by listing explicitly the triads which are supposed to be true in each case.

Tables II and III show how these examples are used in establishing complete independence, a plus sign indicating that a postulate holds, a minus sign, that it fails. For example, in connection with Set (6) we need to exhibit a system $(K, R)$ having the record

\begin{tabular}{|rrrrrr|}
\hline $\mathrm{D}$ & $\mathrm{A}$ & $\mathrm{B}$ & $\mathrm{C}$ & 2 & 4 \\
\hline+ & + & - & + & - & - \\
\hline
\end{tabular}

Turning to Table II, record No. 12, we see that Example 72 is the system required.

To obtain a similar record with the $\mathrm{D}+$ changed to $\mathrm{D}-$, we have only to change Example 72 to Example $72 \mathrm{~d}$.

It will be noted that postulate $\mathrm{D}$ (which demands that if $A B C$ is a true triad, then $A, B$, and $C$ shall be distinct) plays a peculiar rôle, since, though it is strictly independent and cannot be omitted, yet it is never used in proving any of the "theorems of deducibility", and its holding or failing does not effect the holding or failing of any of the other postulates.

\footnotetext{
* The proof of the non-existence of these examples was communicated to the writer by Professor Huntington, who showed that the simpler example, satisfying A, B, C, D, 8 and violating 3, can be found only when $n=4$ or $n=5$, and does not exist when $n=6$ or $n>6$ (where $n$ is the number of elements in the class $\mathrm{K}$ ). This is an altogether unexpected state of affairs, since in previous discussions of complete independence, an increase in the number of elements has always increased (instead of diminishing) the likelihood of finding an example of any desired type. It also suggests a wider inquiry into the validity of the "Lemmas on non-deducibility" (pp. 272-74 of the second paper cited) when $n$ is greater than four. For example, although postulate 3 is in general not deducible from postulates A, B, C, D, 8 (see Lemma 3.1), yet if we add the further condition that the class $\mathrm{K}$ shall contain six or more elements, then postulate 3 can be so deduced. Again Mr. C. H. Langford has shown that postulate 4, though not deducible from postulates A, B, C, D, 8, alone (see Lemma 4.1), can be deduced from these postulates with the added condition that the class $\mathrm{K}$ shall contain at least five elements. How many other similar instances may exist has not yet been investigated.
} 
TABLE ONE

In Examples 1-129, the class $K$ consists of four elements, 1, 2, 3, 4.

Fx. In Examples 501-518, the class $K$ consists of five elements, 1, 2, 3, 4, 5.

$\begin{array}{lllllllll}1 & 123 & 124 & 134 & 234 & 321 & 421 & 431 & 432\end{array}$

$\begin{array}{lllllllll}2 & 123 & 124 & 134 & 321 & 324 & 421 & 423 & 431\end{array}$

$\begin{array}{lllllllll}3 & 123 & 143 & 214 & 234 & 321 & 341 & 412 & 432\end{array}$

$\begin{array}{lllllllll}4 & 123 & 124 & 134 & 243 & 321 & 342 & 421 & 431\end{array}$

$\begin{array}{lllllllllllll}5 & 123 & 124 & 132 & 134 & 231 & 234 & 321 & 324 & 421 & 423 & 431 & 432\end{array}$

$\begin{array}{lllllllllll}6 & 123 & 124 & 132 & 134 & 231 & 234 & 321 & 421 & 431 & 432\end{array}$

$\begin{array}{lllllllllll}7 & 123 & 132 & 214 & 231 & 234 & 314 & 321 & 412 & 413 & 432\end{array}$

$\begin{array}{lllllllllll}8 & 123 & 124 & 132 & 134 & 231 & 243 & 321 & 342 & 421 & 431\end{array}$

$\begin{array}{lllll}9 & 123 & 124 & 321 & 421\end{array}$

$\begin{array}{lllll}10 & 123 & 142 & 241 & 321\end{array}$

$\begin{array}{lllll}11 & 123 & 214 & 321 & 412\end{array}$

$\begin{array}{lllllll}12 & 123 & 142 & 241 & 314 & 321 & 413\end{array}$

$\begin{array}{lllll}13 & 123 & 132 & 231 & 321\end{array}$

$\begin{array}{lllllll}14 & 123 & 124 & 132 & 231 & 321 & 421\end{array}$

$\begin{array}{lllllll}15 & 123 & 132 & 231 & 234 & 321 & 432\end{array}$

$\begin{array}{lllllll}16 & 123 & 132 & 214 & 231 & 321 & 412\end{array}$

$\begin{array}{llllll}17 & 123 & 124 & 134 & 234\end{array}$

$\begin{array}{lllll}18 & 123 & 124 & 134 & 324\end{array}$

$\begin{array}{lllll}19 & 123 & 124 & 134 & 342\end{array}$

$\begin{array}{llllll}20 & 123 & 234 & 341 & 421\end{array}$

$\begin{array}{lllllll}21 & 123 & 124 & 132 & 134 & 234\end{array}$

$\begin{array}{llllll}22 & 123 & 124 & 132 & 342 & 431\end{array}$

$\begin{array}{lllllll}23 & 123 & 124 & 132 & 243 & 431\end{array}$

$\begin{array}{lllllll}24 & 123 & 124 & 132 & 324 & 413\end{array}$

$25 \quad 123 \quad 124$

$\begin{array}{lll}26 & 123 & 243\end{array}$

$\begin{array}{lll}27 & 123 & 234\end{array}$

$\begin{array}{llll}28 & 123 & 241 & 413\end{array}$

$\begin{array}{llll}29 & 123 & 124 & 132\end{array}$

$\begin{array}{llll}30 & 123 & 132 & 243\end{array}$

$\begin{array}{llll}31 & 123 & 132 & 234\end{array}$

$\begin{array}{llll}32 & 123 & 132 & 412\end{array}$

$\begin{array}{lllllllll}33 & 123 & 124 & 234 & 314 & 321 & 413 & 421 & 432\end{array}$

$\begin{array}{lllllllllll}34 & 123 & 132 & 142 & 143 & 231 & 241 & 243 & 321 & 341 & 342\end{array}$

$\begin{array}{lllllllllll}35 & 123 & 124 & 132 & 143 & 231 & 234 & 321 & 341 & 421 & 432\end{array}$

$\begin{array}{lllll}36 & 132 & 142 & 231 & 241\end{array}$

$\begin{array}{lllllll}37 & 123 & 143 & 214 & 321 & 341 & 412\end{array}$ 


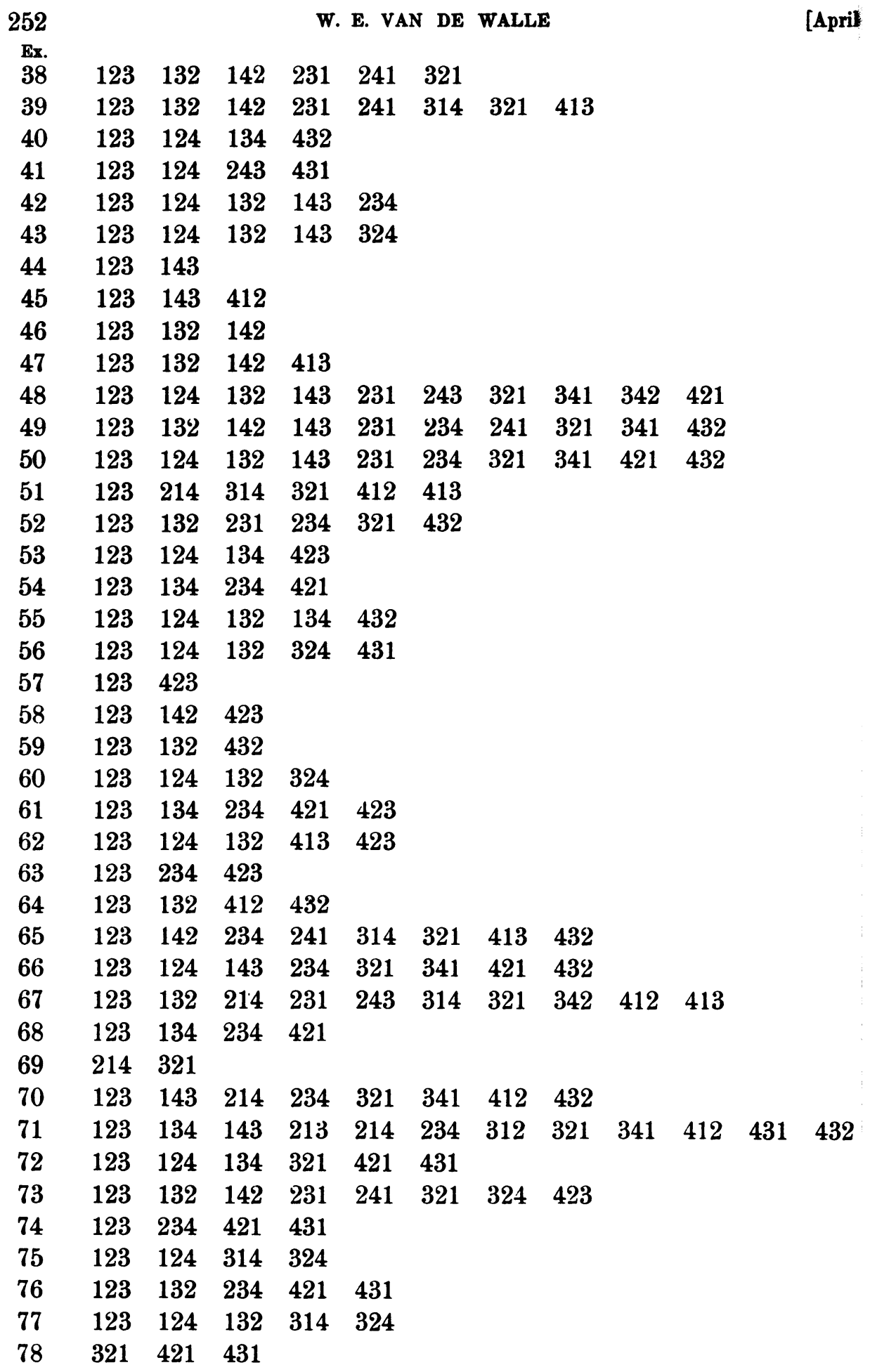




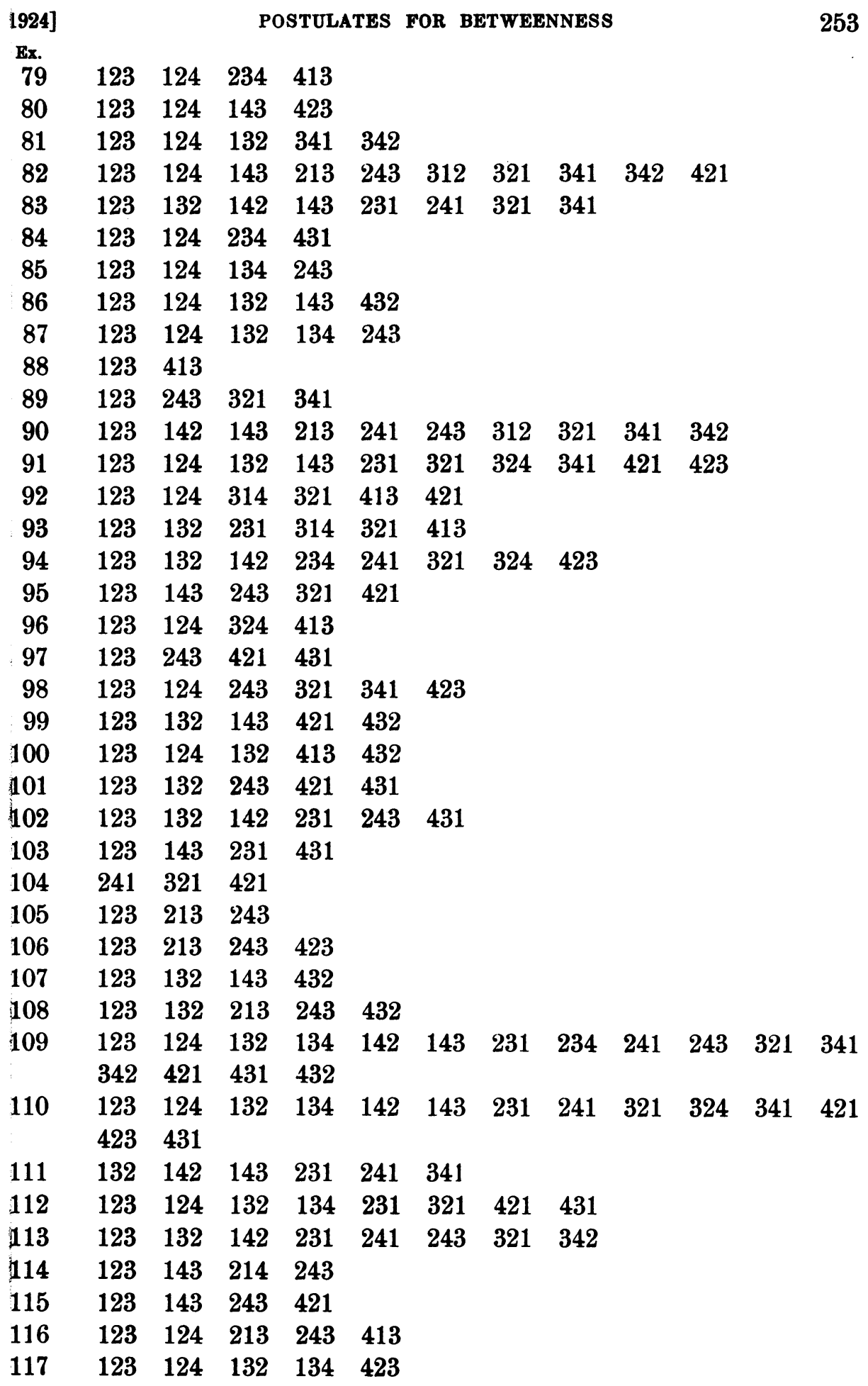


Ex.

$\begin{array}{llllll}118 & 123 & 124 & 132 & 314 & 432\end{array}$

$\begin{array}{llllllll}119 & 123 & 132 & 142 & 241 & 243 & 341\end{array}$

$\begin{array}{llll}120 & 123 & 243 & 423\end{array}$

$\begin{array}{lllll}121 & 123 & 132 & 142 & 342\end{array}$

$\begin{array}{llllll}122 & 123 & 132 & 243 & 423\end{array}$

$\begin{array}{llllll}123 & 123 & 132 & 213 & 243 & 423\end{array}$

$\begin{array}{lllll}124 & 123 & 132 & 412 & 423\end{array}$

$\begin{array}{lllllllllllll}125 & 123 & 132 & 142 & 143 & 213 & 231 & 241 & 243 & 312 & 321 & 341 & 342\end{array}$

$\begin{array}{lllllllllll}126 & 123 & 143 & 213 & 214 & 243 & 312 & 321 & 341 & 342 & 412\end{array}$

$\begin{array}{lllllllll}127 & 123 & 132 & 214 & 231 & 234 & 321 & 412 & 432\end{array}$

$\begin{array}{lllllllll}128 & 123 & 124 & 132 & 143 & 231 & 321 & 341 & 421\end{array}$

$\begin{array}{lllll}129 & 123 & 132 & 142 & 432\end{array}$

1d-129d. Same as 1-129, with the addition of the triad 111.

\begin{tabular}{|c|c|c|c|c|c|c|c|c|c|c|c|c|}
\hline \multirow[t]{2}{*}{$\begin{array}{c}\text { Ex. } \\
501\end{array}$} & 123 & 125 & 134 & 135 & 142 & 145 & 234 & 241 & \multirow[t]{2}{*}{245} & \multirow[t]{2}{*}{321} & \multirow[t]{2}{*}{325} & \multirow[t]{2}{*}{345} \\
\hline & 431 & 432 & 521 & 523 & 531 & 541 & 542 & 543 & & & & \\
\hline 502 & 123 & 125 & 234 & 321 & 432 & 521 & & & & & & \\
\hline 03 & 123 & 153 & 243 & 321 & 342 & 351 & & & & & & \\
\hline \multirow[t]{2}{*}{504} & 123 & 125 & 143 & 145 & 214 & 234 & 254 & 315 & 321 & 325 & 341 & 345 \\
\hline & 412 & 432 & 452 & 513 & 521 & 523 & 541 & 543 & & & & \\
\hline \multirow[t]{2}{*}{505} & 123 & 124 & 145 & 153 & 215 & 235 & 243 & 245 & 314 & 321 & 342 & 345 \\
\hline & 351 & 413 & 421 & 512 & 532 & 541 & 542 & 543 & & & & \\
\hline \multirow[t]{2}{*}{506} & 123 & 125 & 143 & 145 & 213 & 214 & 215 & 243 & 245 & 312 & 315 & 321 \\
\hline & 325 & 341 & 342 & 345 & 412 & 512 & 513 & 521 & 523 & 541 & 542 & 543 \\
\hline 07 & 123 & 143 & 321 & 325 & 341 & 523 & & & & & & \\
\hline 08 & 123 & 153 & 243 & 321 & 342 & 351 & & & & & & \\
\hline 09 & 123 & 124 & 153 & 243 & 321 & 342 & 351 & 421 & & & & \\
\hline 10 & 123 & 132 & 231 & 245 & 321 & 542 & & & & & & \\
\hline 311 & 123 & 143 & 235 & 321 & 325 & 341 & 523 & 532 & & & & \\
\hline \multirow[t]{2}{*}{512} & 123 & 125 & 135 & 143 & 145 & 214 & 234 & 254 & 321 & 325 & 341 & 345 \\
\hline & 412 & 432 & 452 & 521 & 523 & 531 & 541 & 543 & & & & \\
\hline \multirow[t]{2}{*}{513} & 123 & 125 & 134 & 142 & 145 & 153 & 241 & 243 & 245 & 321 & 325 & 342 \\
\hline & $34 \check{~}$ & 351 & 431 & 521 & 523 & 541 & 542 & 543 & & & & \\
\hline \multirow[t]{2}{*}{514} & 123 & 124 & 134 & 153 & 154 & 215 & 235 & 243 & 254 & 321 & 342 & 351 \\
\hline & 354 & 421 & 431 & 451 & 452 & 453 & 512 & 532 & & & & \\
\hline 515 & 123 & 153 & 243 & 321 & 325 & 342 & 351 & 523 & & & & \\
\hline 516 & 123 & 132 & 142 & 143 & 231 & 234 & 241 & 321 & 341 & 415 & 432 & 514 \\
\hline \multirow[t]{2}{*}{517} & 123 & 125 & 143 & 145 & 153 & 214 & 234 & 254 & 321 & 325 & 341 & 345 \\
\hline & 351 & 412 & 432 & 452 & 521 & 523 & 541 & 543 & & & & \\
\hline & 123 & 153 & 243 & 321 & 325 & 342 & 351 & 523 & & & & \\
\hline
\end{tabular}


TABLE TWO

\begin{tabular}{|c|c|c|c|c|c|c|c|c|c|c|}
\hline \multirow[t]{9}{*}{ Rec. } & \multicolumn{2}{|r|}{ Postulates } & \multicolumn{8}{|c|}{ Independent Sets } \\
\hline & & $\begin{array}{lllll}\mathbf{A} & \mathbf{B} & \mathbf{C} & \mathbf{1} & \mathbf{2}\end{array}$ & (1) & & & & & & & \\
\hline & & $\begin{array}{llllll}\mathbf{A} & \mathbf{B} & \mathbf{C} & \mathbf{1} & \mathbf{5} \\
\end{array}$ & & (2) & & & & & & \\
\hline & $\mathbf{D}$ & $\begin{array}{lllll}A & B & C & 1 & 6 \\
\end{array}$ & & & (3) & & & & & \\
\hline & D & $\begin{array}{lllll}\text { A } & \text { B } & \text { C } & \mathbf{1} & \mathbf{7}\end{array}$ & & & & (4) & & & & \\
\hline & $\mathrm{D}$ & 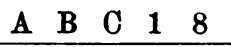 & & & & & (5) & & & \\
\hline & & $\begin{array}{lllll}\mathbf{A} & \mathbf{B} & \mathbf{C} & \mathbf{2} & \mathbf{4} \\
\end{array}$ & & & & & & $(6)$ & & \\
\hline & D & 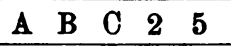 & & & & & & & (7) & \\
\hline & $\mathbf{D}$ & $\begin{array}{lllll}\mathbf{A} & \mathbf{B} & \mathbf{C} & \mathbf{3} & \mathbf{5} \\
\end{array}$ & & & & & & & & (8) \\
\hline 1 & & $+t+t+$ & 1 & 1 & 1 & 1 & 1 & 1 & 1 & 1 \\
\hline 2 & + & ++++- & 2 & 2 & 2 & 2 & 2 & 70 & 70 & 2 \\
\hline 3 & & $+t+-t$ & 3 & 33 & 4 & 4 & 65 & 2 & 33 & 33 \\
\hline 4 & + & +++-- & 4 & 4 & 33 & 501 & 66 & 4 & 4 & 4 \\
\hline $\mathbf{5}$ & & ++-++ & 5 & 6 & 48 & 48 & 48 & 7 & 7 & 6 \\
\hline 6 & + & ++-+- & 6 & 34 & 6 & 6 & 6 & 71 & 71 & 82 \\
\hline 7 & & ++--+ & 7 & 8 & 49 & 8 & 67 & 6 & 6 & 8 \\
\hline 8 & + & ++--- & 8 & 35 & 50 & 35 & 8 & 8 & 35 & 35 \\
\hline 9 & & $+-t+t$ & 9 & 9 & 10 & 10 & 10 & 9 & 9 & 9 \\
\hline 10 & + & +-++- & 10 & 36 & 9 & 9 & 9 & 36 & 36 & 36 \\
\hline 11 & + & +-+-+ & 11 & 11. & 11 & 11 & 11 & 10 & 10 & 10 \\
\hline 12 & + & +-+-- & 12 & 37 & 51 & 502 & 51 & 72 & 72 & 503 \\
\hline 13 & + & +--++ & 13 & 14 & 38 & 38 & 38 & 15 & 15 & 15 \\
\hline 14 & + & +--+- & 14 & 38 & 14 & 14 & 14 & 73 & 73 & 83 \\
\hline 15 & + & +---+ & 15 & 16 & 16 & 16 & 16 & 14 & 14 & 14 \\
\hline 16 & + & $+---\cdots$ & 16 & 39 & 52 & 15 & 15 & 38 & 38 & 38 \\
\hline 17 & + & $-t+t+$ & 17 & 17 & 17 & 17 & 17 & 17 & 17 & 17 \\
\hline 18 & + & -+++- & 18 & 40 & 53 & 53 & 53 & 74 & 40 & 40 \\
\hline 19 & + & -++-+ & 19 & 41 & 19 & 19 & 19 & 18 & 79 & 84 \\
\hline 20 & + & -++-- & 20 & 19 & 54 & 61 & 68 & 75 & 80 & 85 \\
\hline 21 & + & -+-++ & 21 & 21 & 21 & 21 & 21 & 21 & 21 & 21 \\
\hline 22 & + & -+-+- & 22 & 42 & 55 & 55 & 55 & 76 & 42 & 86 \\
\hline 23 & + & -+--+ & 23 & 23 & 23 & 23 & 23 & 22 & 81 & 87 \\
\hline 24 & + & -+--- & 24 & 43 & 56 & 62 & 56 & 77 & 62 & 42 \\
\hline 25 & + & $--t+t$ & 25 & 25 & 25 & 25 & 25 & 25 & 25 & 25 \\
\hline 26 & + & --++- & 26 & 44 & 57 & 57 & 57 & 44 & 44 & 44 \\
\hline 27 & + & --+-+ & 27 & 27 & 27 & 27 & 69 & 26 & 26 & 88 \\
\hline 28 & + & --+-- & 28 & 45 & 58 & 63 & 58 & 78 & 78 & 89 \\
\hline 29 & + & ---++ & 29 & 29 & 29 & 29 & 29 & 29 & 29 & 29 \\
\hline 30 & + & ---+- & 30 & 46 & 59 & 59 & 59 & 46 & 46 & 46 \\
\hline 31 & + & ----+ & 31 & 32 & 32 & 32 & 32 & 32 & 32 & 32 \\
\hline 32 & + & $---\cdots$ & 32 & 47 & 60 & 64 & 60 & 47 & 47 & 47 \\
\hline
\end{tabular}


TABLE THREE

\begin{tabular}{|c|c|c|c|c|}
\hline Rec. & Postulates & Indep & enden & t Sets \\
\hline & D A A & (9) & & \\
\hline & $\begin{array}{llllllll} & \mathrm{D} & \mathrm{A} & \mathrm{B} & \mathrm{C} & \mathbf{3} & 4 & 7\end{array}$ & & (10) & \\
\hline & D A & & & (11) \\
\hline 1 & $+t+t+t$ & 1 & 1 & 1 \\
\hline 2 & +++++- & 2 & 2 & 2 \\
\hline 3 & $+t+t+-t$ & 3 & 3 & 3 \\
\hline 4 & +++++-- & 504 & 512 & 517 \\
\hline 5 & $++t+-+t$ & 65 & 65 & 65 \\
\hline 6 & ++++-+ & 33 & 513 & 33 \\
\hline 7 & $+t++-t$ & 4 & 4 & - \\
\hline 8 & ++++-- & 505 & 514 & 4 \\
\hline 9 & $+t+-t+t$ & 90 & 109 & 125 \\
\hline 10 & +++-++- & 6 & 6 & 6 \\
\hline 11 & $++t-+-t$ & 82 & 82 & 82 \\
\hline 12 & +++-+-- & 506 & 110 & 110 \\
\hline 13 & $+++-t+$ & 48 & 48 & 48 \\
\hline 14 & +++--+- & 91 & 91 & 91 \\
\hline 15 & +++--+ & 8 & 8 & 126 \\
\hline 16 & $+++-\cdots$ & 35 & 35 & 8 \\
\hline 17 & $++-t+t$ & 11 & 11 & 11 \\
\hline 18 & ++-+++- & 9 & 9 & 9 \\
\hline 19 & ++-++-+ & 36 & 36 & 36 \\
\hline 20 & ++-++-- & 507 & 111 & 72 \\
\hline 21 & ++-+-+ & 10 & 10 & 10 \\
\hline 22 & ++-+-+- & 92 & 92 & 92 \\
\hline 23 & ++-+--+ & 508 & 508 & 508 \\
\hline 24 & $++-+-\cdots$ & 509 & 515 & 518 \\
\hline 25 & ++--++ & 510 & 510 & 510 \\
\hline 26 & ++--+- & 15 & 15 & 15 \\
\hline 27 & ++--+-+ & 83 & 516 & 83 \\
\hline 28 & ++--+- & 511 & 112 & 127 \\
\hline 29 & ++--++ & 93 & 93 & 93 \\
\hline 30 & ++--+- & 14 & 14 & 14 \\
\hline 31 & ++---+ & 38 & 38 & 38 \\
\hline 32 & $++\cdots-\cdots$ & 94 & 113 & 128 \\
\hline 33 & +-++++ & 17 & 17 & 17 \\
\hline 34 & +-+++-1 & 53 & 53 & 53 \\
\hline
\end{tabular}

\begin{tabular}{|c|c|c|c|c|}
\hline Rec. & Postulates & Indep & (2) & \\
\hline & D A $A$ B & (9) & & \\
\hline & $\begin{array}{llllllll}\text { D } & \text { A } & \text { B } & \text { C } & 3 & 4 & 7\end{array}$ & & (10) & \\
\hline & $\begin{array}{llllllll}D & A & B & C & 3 & 4 & 8\end{array}$ & & & (11) \\
\hline 35 & $+-++-t$ & 75 & 114 & \\
\hline 36 & +-+++-- & 95 & 115 & \\
\hline 37 & +-++-++ & 85 & 85 & \\
\hline 38 & +-++-+- & 96 & 96 & \\
\hline 39 & $+-++-t$ & 97 & 97 & 98 \\
\hline 40 & +-++-- & 98 & 116 & 116 \\
\hline 41 & $+-+-++t$ & 21 & 21 & \\
\hline 42 & +-+-+- & $\mathbf{5 5}$ & 117 & \\
\hline 43 & +-+-+-+ & 62 & 77 & 76 \\
\hline 44 & +-+-+-- & 99 & 62 & 62 \\
\hline 45 & +-+--++ & 87 & 87 & 87 \\
\hline 46 & +-+--+- & 100 & 118 & 100 \\
\hline 47 & +-+--+ & 101 & 101 & 101 \\
\hline 48 & +-+--- & 102 & 119 & 102 \\
\hline 49 & +--+++ & 25 & 25 & 25 \\
\hline 50 & +--++- & 57 & 57 & 57 \\
\hline 51 & +--++-+ & 44 & 44 & 44 \\
\hline 52 & +--++-- & 103 & 78 & 78 \\
\hline 53 & +--+-++ & 89 & 88 & 88 \\
\hline 54 & +--+-+- & 104 & 120 & 120 \\
\hline 55 & +--+--+ & 105 & 105 & 105 \\
\hline 56 & +--+-- & 106 & 106 & 106 \\
\hline 57 & +--+++ & 29 & 29 & 29 \\
\hline 58 & $+\cdots-++-$ & 59 & 59 & 59 \\
\hline 59 & $t--+-+$ & 46 & 46 & 46 \\
\hline 60 & +---+-- & 107 & 121 & 129 \\
\hline 61 & +---++ & 32 & 32 & 32 \\
\hline 62 & +----+ & 124 & 122 & 124 \\
\hline 63 & $+---\cdots+$ & 47 & 47 & 47 \\
\hline 64 & +----- & 108 & 123 & 123 \\
\hline
\end{tabular}

Records No. 65-128 are the same as records No. 1-64 with the $\mathrm{D}+$ changed to $\mathrm{D}$ - , and the letter " $d$ " added to each example-number.

HARVARD UNIVERSITY,

Cambridge, Mass. 